\title{
Powering In-body Nanosensors with Ultrasounds
}

\author{
Michael Donohoe, Student Member, IEEE, Sasitharan Balasubramaniam, Senior Member, IEEE, \\ Brendan Jennings, Member, IEEE and Josep Miquel Jornet, Member, IEEE
}

\begin{abstract}
Embedded nanosensors will be a key feature of emerging medical monitoring systems. Power for these sensors could be harvested from ultrasonic vibrations generated by portable miniature sources and converted to electrical energy by piezoelectric nanowires. This letter analyses the frequency and intensity of ultrasounds required to power an embedded nanosensor subject to medical safety limits, absorption by human tissue and reflection from interfaces. We calculate input and output power at different levels of energy conversion efficiency. Our analysis suggests that ultrasounds can be a viable source for energy harvesting of in-body nanosensors.
\end{abstract}

Index Terms-Nanosensors, Energy Harvesting, Ultrasound.

\section{INTRODUCTION}

The Internet of Bio-Nano Things [1] aims to interconnect heterogeneous molecular communication networks within the human body to the outside world through a bio-cyber interface. However there are major challenges to overcome in the deployment of networks of nano-devices within a living biological environment. On the one hand the miniature size of the devices and the medium in which they are deployed can change their material properties. On the other hand the very high frequencies that nano-devices need for communication show limited penetration through biological tissue. Moreover, network components must have sufficient power for data collection, computation and transmission [2]. One method of power provision is energy harvesting from external sources (electromagnetic, vibrational, or thermal). In this letter we propose the use of energy harvesting electromagnetic (EM) nanosensors (which we refer to as nanosensors) operating in the Terahertz, infra-red or optical wavebands to enable communications. Our proposed scenario is illustrated in Figure 1, where a nanosensor is encapsulated in a bio-compatible artificial cell and is embedded into human tissue. Ultrasounds from a portable external source then induce vibrations in piezoelectric nanowires in the nanosensor to harness energy for the device. The powering of sensors embedded in human tissue by ultrasounds was investigated by Ozeri and

M. Donohoe and B. Jennings are with the Telecommunications Software \& Systems Group (TSSG), Waterford Institute of Technology, Ireland. S Balasubramaniam is with the Tampere University of Technology, Finland J. M. Jornet is with the University at Buffalo, State University of New York, USA.Email: mdonohoe@tssg.org, sasi.bala@tut.fi, bjennings@tssg.org, jmjornet@buffalo.edu.

This work is supported by the Academy of Finland FiDiPro programme for the project "Nanocommunications Networks" 2012 - 2016, and the Finnish Academy Research Fellow programme under Project no. 284531. It is also partly funded by the Irish Higher Education Authority under the Programme for Research in Third Level Institutions (PRTLI) cycle 5, which is co-funded by the European Regional Development Fund (ERDF), via the Telecommunications Graduate Initiative, and by Science Foundation Ireland via the CONNECT research centre (grant no. 13/RC/2077), which is cofunded by the European Regional Development Fund (ERDF).

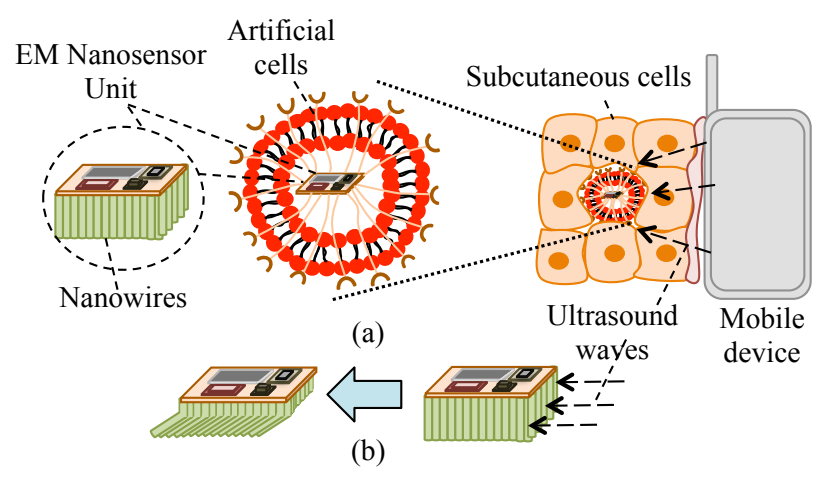

Fig. 1: Scenario of Ultrasounds (a) propagating from an external device through skin and fat cells to embedded EMbased nanosensor, and (b) bending piezoelectric nanowires.

Schmilovitz [3] but this was at a macro $\left(\mathrm{cm}^{2}\right)$ and not at the nano scale. Wang et al used ultrasounds to power a 2 $\mathrm{mm}^{2}$ nanogenerator immersed in water [4] and biofluid [5]. The ultrasound excitation at $41 \mathrm{kHz}$ produced measurable voltage and current but the intensity of the ultrasound and the energy conversion efficiency were not stated. The balancing of energy production and energy requirements of a $1000 \mu \mathrm{m}^{2}$ AC nanogenerator was examined by Jornet and Akyildiz [6]. A cyclic vibrational force of $50 \mathrm{~Hz}$ was applied in the model and the harvested energy per cycle was stored in a nanocapacitor with a maximum capacity of $800 \mathrm{pJ}$. The model showed how this level of energy could be used for basic data transmission.

This letter models the novel use of a miniature external ultrasound source to power an embedded nanosensor. We calculate the energy available for harvesting at the nanosensor and compute the potential power output. Energy-harvesting principles using ultrasounds are outlined in $\S$ II. Our modelling of harvested energy is discussed in $\S I I I$, the power output is analysed in $\S \mathrm{IV}$ and our conclusions and future work are presented in $\S \mathrm{V}$.

\section{NANOSENSOR Energy HaRvesting}

An energy harvesting nanosensor will include a nanogenerator with nanowires that convert induced vibrations from an external ultrasound source to electrical energy (Figure 1).

\section{A. Ultrasounds as an Energy Source}

Ultrasounds are generated when an electric field is applied to an array of piezoelectric crystals located on a transducer surface. Electrical stimulation causes mechanical distortion of the crystals resulting in vibration and production of sound waves. In our study we consider ultrasound frequencies in the 
range from $50 \mathrm{kHz}$ to $1 \mathrm{MHz}$. We use a maximum ultrasound intensity of $720 \mathrm{~mW} / \mathrm{cm}^{2}$ in line with medical recommendations [7]. We model a miniature portable ultrasound source as emitting a narrow beam $\left(\mathrm{mm}^{2}\right)$ into human tissue with no spreading. The ultrasound intensity arriving at the nanosensor depends on the power output of the ultrasound source, the distance from the nanosensor and the attenuation of the beam by any medium in the path. The attenuation is caused by (i) the absorption of energy by the tissue (through molecular vibrations and relaxation) and (ii) the reflection of energy at tissue interfaces. We do not include in this model other factors that can contribute to attenuation. These include cases where the nanosensor maybe tilted with respect to the beam or where the nanosensor is deformed and displaced by tissue movements.

For our proposed approach, three different power intensities need to be modelled: (i) the initial intensity emanating from the ultrasound source; (ii) the ultrasound intensity entering the nanogenerator following penetration through tissue layers; and (iii) the piezoelectric power intensity emerging from the nanogenerator.

\section{B. Ultrasound Absorption in Human Tissue}

The parameters we use for ultrasound transmission in the human body are based on those of medical imaging [7]. First we consider energy absorption in the tissue layer. If the initial intensity is $I_{o}$ then the intensity $I_{d}$ for a beam of frequency $f$ $\mathrm{MHz}$ at a depth of $d \mathrm{~cm}$ is represented as:

$$
I_{d}=I_{o} 10^{-(\alpha f d / 10)}
$$

where the absorption coefficient $\alpha$, expresses the power loss and has a value of $0.6 \mathrm{~dB} / \mathrm{cm} / \mathrm{MHz}$ for skin/fat. The reduction in intensity at different frequencies and skin/fat depths up to $10 \mathrm{~cm}$ is shown in Figure 2. Higher frequencies are more strongly absorbed than lower frequencies and the graph shows that a $1 \mathrm{MHz}$ beam has lost half of its intensity $(3 \mathrm{~dB})$ at a skin/fat depth of $5 \mathrm{~cm}$.

Acoustic reflections at tissue interfaces (e.g., between fat and muscle) are caused by differences in acoustic impedance (the density multiplied by the speed of sound). The unit of acoustic impedance is the Rayl $\left(\mathrm{kg} . \mathrm{s}^{-1} \cdot \mathrm{m}^{-2}\right)$. If two materials have acoustic impedances $Z_{1}$ and $Z_{2}$ then the ratio between incident intensity $I_{o}$ and reflected intensity $I_{r}$ is represented as [7]:

$$
\frac{I_{r}}{I_{o}}=\frac{\left(Z_{2}-Z_{1}\right)^{2}}{\left(Z_{2}+Z_{1}\right)^{2}}
$$

The acoustic impedance of air is 429 Rayl while that of skin/fat is 1.4 MRayl. Therefore, the reflection at an air/human tissue interface would result in up to $99 \%$ of the ultrasound being reflected because of the large difference in the acoustic impedance [7]. Consequently, there should be no air gap between an ultrasound transducer and human tissue. In our model we assume only one type of tissue (skin/fat) between the source and nanosensor with no air gap. For maximum power transfer, the absorption and the acoustic impedance of the artificial cell membrane and substrate should match the

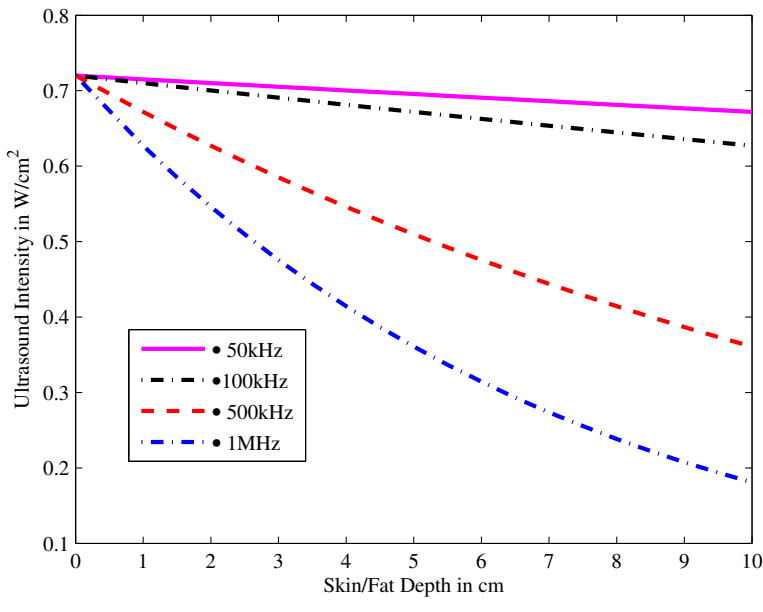

Fig. 2: Plot of Ultrasound Intensity vs Skin/Fat Depth. Initial intensity is $720 \mathrm{~mW} / \mathrm{cm}^{2}$.

characteristics of body tissue as closely as possible (e.g., using polymer-based materials).

\section{Modelling Ultrasound EnERgy Harvesting}

The nanogenerator must convert incident ultrasounds into mechanical vibrations and then into piezoelectric energy. The use of Zinc Oxide $(\mathrm{ZnO})$ nanowires for energy harvesting was proposed by Wang and Song [8]. The nanowires in this type of DC nanogenerator are fixed at one end to a substrate while the other end is free and can bend. Systematically bending the nanowires produces current and voltage amounts that are collected by an electrode. The maximum potential at the nanowires surface is directly proportional to the bending and inversely proportional to the length-to-diameter aspect ratio. In [8] the bending is performed by an atomic force microscope $(A F M)$ tip. In our model the nanowire bending is driven by ultrasound. The nanogenerator power harvesting depends on: (i) the amount of bending the nanowires are subjected to; (ii) the bending events per second (frequency); and (iii) the nanowires per unit area (density). The energy per cycle of the ultrasounds will determine the amount of bending, while the frequency of the ultrasounds will determine the quantity of bends per second.

\section{A. Bending a Nanowire}

The following analysis of the energy needed to bend a nanowire is based on work by Wang and Song [8] for a $\mathrm{ZnO}$ nanowire DC nanogenerator. A nanowire can be modelled as a thin cylindrical rod with a specific modulus of elasticity (Young's modulus). Bending a nanowire requires the application of a force and this is countered by the elasticity of the nanowire. If a constant force $F$ is applied until a maximum bending before discharge, denoted $y_{m}$, is achieved, then the balance of forces is represented as:

$$
F=\frac{3 Y I y_{m}}{L^{3}}
$$




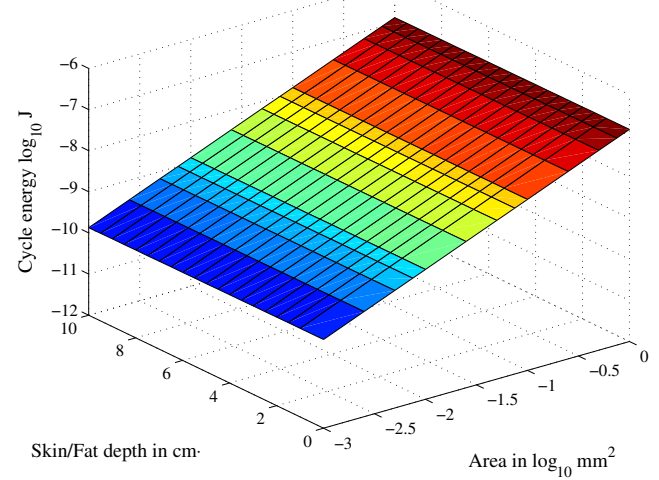

(a) $50 \mathrm{kHz}$ ultrasound wave.

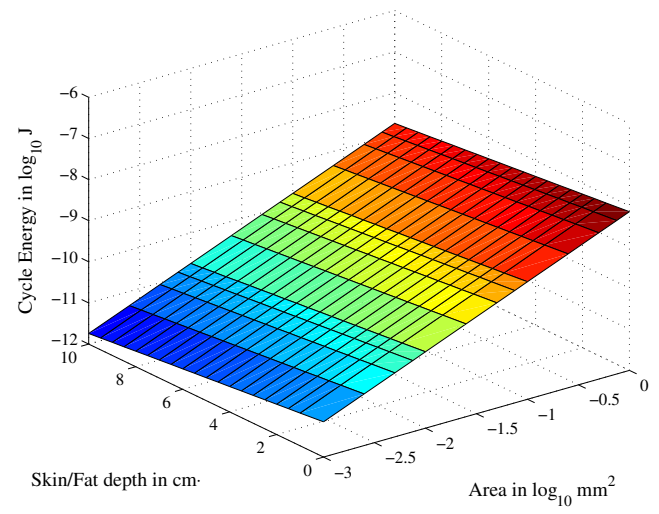

(b) $1 \mathrm{MHz}$ ultrasound wave

Fig. 3: Energy per cycle at $50 \mathrm{kHz}$ (a) and $1 \mathrm{MHz}$ (b) against skin/fat depth and varying nanogenerator area.

where $Y$ is the elastic modulus of a nanowire, $I$ is the area moment of inertia and $L$ is the length. This equation can be used to calculate the maximum bending that corresponds to a specific applied force. The energy (work), denoted $\Delta E$, required to bend the nanowire by an amount $y_{m}$ is represented as:

$$
\Delta E=\frac{3 Y I y_{m}^{2}}{2 L^{3}}
$$

Examples of calculated values for force, displacement and work for bending a nanowire that is $50 \mathrm{~nm}$ in diameter and $600 \mathrm{~nm}$ long using an AFM tip are shown in Table I. The work required for bending is of the order of femtojoules and the magnitude of bending is sufficient to deliver a piezoelectric energy output.

TABLE I: Force, displacement and work for bending a nanowire.

\begin{tabular}{lll}
\hline Force $(n N)$ & Displacement $(\mathrm{nm})$ & Work $(\mathrm{fJ})$ \\
\hline 60 & 109 & 3.274 \\
80 & 146 & 5.821 \\
90 & 164 & 7.36 \\
100 & 182 & 9.09 \\
\hline
\end{tabular}

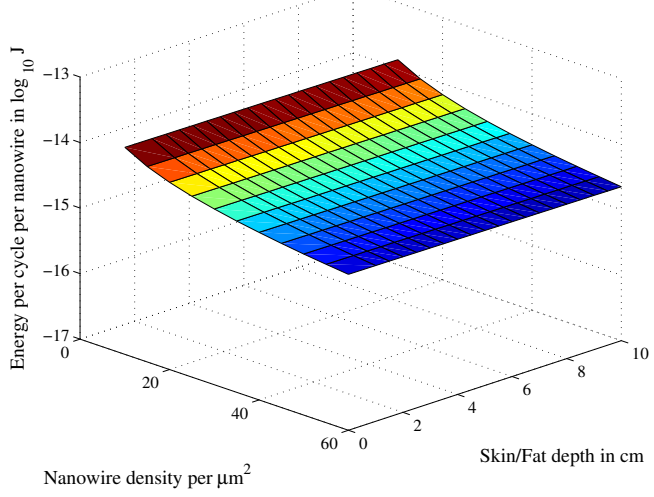

(a) $50 \mathrm{kHz}$ ultrasound wave.

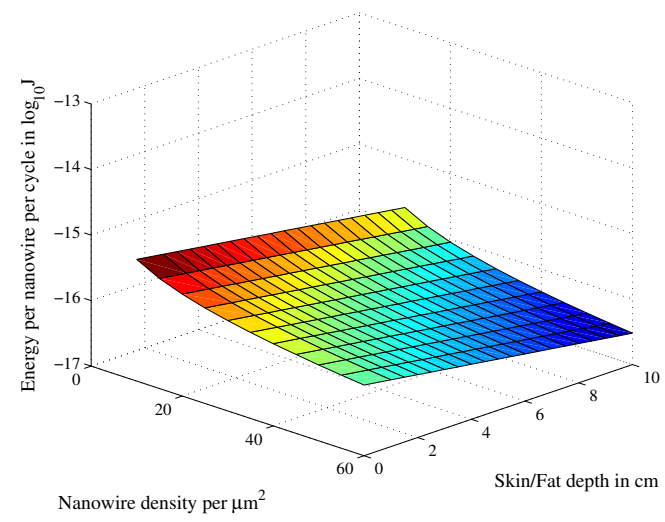

(b) $1 \mathrm{MHz}$ ultrasound wave.

Fig. 4: Energy per cycle at $50 \mathrm{kHz}$ (a) and $1 \mathrm{MHz}$ (b) against skin/fat depth and varying nanowire density.

\section{B. Ultrasound Cycle Energy}

We need to compare the work levels for bending a nanowire in Table I with the energy that can be delivered to a nanowire by ultrasounds. A plot of delivered energy per vibrational cycle at $50 \mathrm{kHz}$ and $1 \mathrm{MHz}$ for a range of nanogenerator areas and different skin/fat depths is shown in Figure 3. The nanowire density is set at 20 per $\mu \mathrm{m}^{2}$, the initial input intensity is fixed at $720 \mathrm{~mW} / \mathrm{cm}^{2}$, and the intensity at different depths is calculated using (1). At a fixed density, the energy per nanowire per cycle is independent of the area of the nanogenerator. At 50 $\mathrm{kHz}$ the energy level is from $7.1 \mathrm{fJ}$ to $6.7 \mathrm{fJ}$ at $1 \mathrm{~cm}$ and $10 \mathrm{~cm}$ depth, respectively. The energy per cycle per nanowire at $1 \mathrm{MHz}$ is initially over 20 times lower than at $50 \mathrm{kHz}$ $(0.03 \mathrm{fJ})$ and decreases more rapidly with depth. A second plot of energy per cycle per nanowire for a fixed area (1000 $\mu \mathrm{m}^{2}$ ), but with varying nanowire densities and skin/fat depths is shown in Figure 4. Higher nanowire densities will reduce the energy absorbed per nanowire for a fixed area. At $50 \mathrm{kHz}$ the energy per nanowire per cycle drops below $6 \mathrm{fJ}$ at these values (Figure 4: Density 20, Skin/Fat depth $10 \mathrm{~cm}$ ). At 1 $\mathrm{MHz}$ the energy per nanowire is lower than $1 \mathrm{fJ}$ at all densities and depths. Both plots show that for a fixed input intensity, an 
ultrasound frequency of $50 \mathrm{kHz}$ will deliver more than $6 \mathrm{fJ}$ per cycle to a nanowire provided the density is equal to or lower than 20 nanowires per $\mu \mathrm{m}^{2}$. This means that the magnitude of $50 \mathrm{kHz}$ ultrasound cycle energies per nanowire are comparable to the bending energies shown in Table I, but the $1 \mathrm{MHz}$ cycle energies are too low to provide sufficient bending.

\section{Power OutPut Analysis}

The total output energy of a nanogenerator depends on factors such as area, piezoelectric efficiency of the nanowires, absorption or reflection of ultrasound within the nanosensor and the fraction of nanowires that contribute to the electrical output. The DC $\mathrm{ZnO}$ nanogenerator in [8] had an output energy per nanowire of approximately $0.05 \mathrm{fJ}$ but this did not use ultrasound. Comparing this output to an input level of between $5.82 \mathrm{fJ}$ (force of $80 \mathrm{nN}$ ) and $9.09 \mathrm{fJ}$ (force of 100 $\mathrm{nN}$ ) per nanowire in Table I suggests a conversion efficiency of between $0.55 \%$ and $0.8 \%$. Therefore a $1000 \mu \mathrm{m}^{2}$ ultrasound harvesting nanogenerator with 20 nanowires per $\mu \mathrm{m}^{2}$ and a work per nanowire of $7 \mathrm{fJ}$ could have a power output of $38.5 \mathrm{nW}$ when a conversion factor of $0.55 \%$ is used. Proposed pulse-based modulation techniques (as described by Jornet and Akyildiz [9]) require average power levels of $1 \mu \mathrm{W}$ to $10 \mu \mathrm{W}$. Spreading the pulses in time by a factor of 100 to 1000 brings the average power back to the $\mathrm{nW}$ level.

\section{CONClusion And Future Work}

We have shown that an external ultrasound source can theoretically supply vibrational energy to an embedded nanosensor that uses the bending of $\mathrm{ZnO}$ nanowires to produce power. The intensity of the ultrasounds must remain within safe medical limits and there must be no air gap between the source and the human skin. In this letter we have analysed the amount of force and work required to bend the nanowires and the quantity of ultrasound energy delivered over varying nanogenerator areas and densities of nanowire with respect to the skin/fat depth. Our proposed approach can lead to new energy harvesting techniques for the Bio-cyber interface of the Internet of BioNano Things. Based on our analysis the nanosensor unit can provide higher $\mu \mathrm{W}$ power levels by: (i) engineering the nanowire density and dimensions for maximum efficiency; (ii) optimising the collection area of the nanogenerator; and (iii) cascading nanogenerators to produce more power. However, a frequency of $50 \mathrm{kHz}$ delivering nanowatts of power may provide enough energy over a number of seconds for the direct operation of the nanosensor without the need for intermediate battery or capacitor storage.

As part of our future work we will model a portable (or onbody) ultrasound transducer device. The overall efficiency of a portable battery-operated device and energy harvesting system will depend on factors such as: (i) DC to AC conversion in the unit (typically 80\% efficiency); (ii) AC electric to ultrasound conversion (typically 90\%); (iii) attenuation in the tissue; (iv) movement of the the nanosensor; (v) piezoelectric nanowire conversion process (less than 1\%); and (vi) the electrical demand of the nanosensor for processing and transmission of data.
Clearly, the greatest proportional reduction will be within the energy harvesting system. An overall efficiency can be computed in the case where the actual function of the nanosensor and its electrical requirement is known.

While we analysed a single nanosensor here, we also intend to study clusters of nanosensors embedded in the body. A cluster can provide greater data collection capability while mitigating the effects of slight movements or changes in orientation of individual nanosensors. The total power supplied to any cluster would still have to fit within safe medical limits. Clusters will improve overall efficiency by ensuring that a greater fraction of the ultrasound power is intercepted and used for harvesting.

\section{REFERENCES}

[1] I. Akyildiz, M. Pierobon, S. Balasubramaniam, and Y. Koucheryavy, "The internet of bio-nano things," IEEE Communications Magazine, vol. 53, no. 3, pp. 32-40, March 2015.

[2] M. Kocaoglu and O. Akan, "Minimum energy channel codes for nanoscale wireless communications," IEEE Transactions on Wireless Communications, vol. 12, no. 4, pp. 1492-1500, April 2013.

[3] S. Ozeri and D. Shmilovitz, "Ultrasonic transcutaneous energy transfer for powering implanted devices," Ultrasonics, vol. 50, no. 6, pp. $556-$ 566, 2010.

[4] X. Wang, J. Song, J. Liu, and Z. L. Wang, "Direct-current nanogenerator driven by ultrasonic waves," Science, vol. 316, no. 5821, pp. 102-105, 2007.

[5] X. Wang, J. Liu, J. Song, and Z. L. Wang, "Integrated nanogenerators in biofluid," Nano Letters, vol. 7, no. 8, pp. 2475-2479, 2007.

[6] J. Jornet and I. Akyildiz, "Joint energy harvesting and communication analysis for perpetual wireless nanosensor networks in the terahertz band,' IEEE Transactions on Nanotechnology, vol. 11, no. 3, pp. 570-580, May 2012.

[7] W. R. Hendee and E. R. Ritenour, Ultrasound Waves. John Wiley \& Sons, Inc., 2003, pp. 303-316.

[8] Z. L. Wang and J. Song, "Piezoelectric nanogenerators based on zinc oxide nanowire arrays," Science, vol. 312, no. 5771, pp. 242-246, 2006.

[9] J. Jornet and I. Akyildiz, "Femtosecond-long pulse-based modulation for terahertz band communication in nanonetworks," IEEE Transactions on Communications, vol. 62, no. 5, pp. 1742-1754, May 2014. 